\title{
Mutations with pathogenic potential in proteins located in or at the composite junctions of the intercalated disk connecting mammalian cardiomyocytes: a reference thesaurus for arrhythmogenic cardiomyopathies and for Naxos and Carvajal diseases
}

\author{
Steffen Rickelt $\cdot$ Sebastian Pieperhoff
}

Received: 31 January 2012 / Accepted: 3 February 2012 / Published online: 27 March 2012

(C) The Author(s) 2012. This article is published with open access at Springerlink.com

\begin{abstract}
In the past decade, an avalanche of findings and reports has correlated arrhythmogenic ventricular cardiomyopathies (ARVC) and Naxos and Carvajal diseases with certain mutations in protein constituents of the special junctions connecting the polar regions (intercalated disks) of mature mammalian cardiomyocytes. These molecules, apparently together with some specific cytoskeletal proteins, are components of (or interact with) composite junctions. Composite junctions contain the amalgamated fusion products of the molecules that, in
\end{abstract}

This work was supported in part by the German Cancer Foundation (Deutsche Krebshilfe, grants 10-2049-Fr1 and 106976 to W.W.F.) and the Federal Ministry for Research and Technology (START-MSC; grant 01GN0942). S.P. thanks, in particular, the German Science Foundation (DFG) for funding of a postdoctoral fellowship (Pi 869/1-1) and the British Heart Foundation (BHF) for subsequent funding within the BHF Centres of Research Excellence (BHF CoRE).

\section{S. Rickelt $(\bowtie)$}

Helmholtz Group for Cell Biology,

German Cancer Research Center (DKFZ),

Im Neuenheimer Feld 581, Building TP4,

69120 Heidelberg, Germany

e-mail: s.rickelt@dkfz.de

S. Rickelt

Progen Biotechnik,

Heidelberg, Germany

S. Pieperhoff $(\bowtie)$

BHF Centre for Cardiovascular Science,

The Queen's Medical Research Institute, University of Edinburgh,

47 Little France Crescent,

EH164TJ Edinburgh, Scotland, UK

e-mail: spieperh@staffmail.ed.ac.uk other cell types and tissues, occur in distinct separate junctions, i.e. desmosomes and adherens junctions. As the pertinent literature is still in an expanding phase and is obviously becoming important for various groups of researchers in basic cell and molecular biology, developmental biology, histology, physiology, cardiology, pathology and genetics, the relevant references so far recognized have been collected and are presented here in the following order: desmocollin-2 (Dsc2, DSC2), desmoglein-2 (Dsg2, DSG2), desmoplakin (DP, DSP), plakoglobin (PG, JUP), plakophilin-2 (Pkp2, PKP2) and some non-desmosomal proteins such as transmembrane protein 43 (TMEM43), ryanodine receptor 2 (RYR2), desmin, lamins $\mathrm{A}$ and $\mathrm{C}$, striatin, titin and transforming growth factor$\beta 3$ (TGF $\beta 3$ ), followed by a collection of animal models and of reviews, commentaries, collections and comparative studies.

Keywords Arrhythmogenic ventricular cardiomyopathy . Carvajal disease - Composite junction - Desmosomes . Intercalated disk $\cdot$ Naxos disease

Table 1 Desmosomal proteins

\begin{tabular}{lll}
\hline Protein & \multicolumn{2}{c}{ References presented in alphabetical and year order } \\
\hline Desmocollin-2 & $\begin{array}{l}\text { Heuser et al. 2006 } \\
\text { Syrris et al. 2006b }\end{array}$ & $\begin{array}{l}\text { Christensen et al. 2010a } \\
\text { Cox et al. 2010 } \\
\text { de Bortoli et al. 2010 } \\
\text { Xu et al. 2010 }\end{array}$ \\
& $\begin{array}{l}\text { Beffagna et al. 2007 } \\
\text { Bhuiyan et al. 2009 } \\
\text { Simpson et al. 2009 }\end{array}$ &
\end{tabular}




Protein References presented in alphabetical and year order

Desmoglein-2 Awad et al. 2006a

Pilichou et al. 2006

Christensen et al. 2010a

Cox et al. 2010

Gehmlich et al. 2010

Syrris et al. 2007

Posch et al. 2008

Yu et al. 2008

Bhuiyan et al. 2009

den Haan et al. 2009

Desmoplakin Norgett et al. 2000

Rampazzo et al. 2002

$\mathrm{Xu}$ et al. 2010

Fressart et al. 2010

Gehmlich et al. 2011b

Kapplinger et al. 2011

Lahtinen et al. 2011

Nakajima et al. 2011

Sato et al. 2011

den Haan et al. 2009

Bolling et al. 2010

Christensen et al. 2010a

Alcalai et al. 2003

Bauce et al. 2005

Norman et al. 2005

Sen-Chowdhry et al. 2005

Cox et al. 2010

Mahoney et al. 2010

Sen-Chowdhry et al. $2010 b$

Xu et al. 2010

Fressart et al. 2010

Norgett et al. 2006

Uzumcu et al. 2006

Yang et al. 2006

Sen-Chowdhry et al. 2007b

Plakoglobin

McKoy et al. 2000

Protonotarios et al. 2001

Protonotarios et al. 2002

Kaplan et al. 2004

Antoniades et al. 2006

Garcia-Gras et al. 2006

$$
\text { Munkholm et al. } 2012
$$

Asimaki et al. 2007

Huang et al. 2008

Plakophilin-2 Gerull et al. 2004

Antoniades et al. 2006

Awad et al. 2006b

Calkins 2006

Dalal et al. 2006

Kannankeril et al. 2006

Nagaoka et al. 2006

Gehmlich et al. 2011a

Lahtinen et al. 2011

Gomes et al. 2012

Asimaki et al. 2009

den Haan et al. 2009

Christensen et al. 2010a

Xu et al. 2010

Asimaki et al. 2011

Gehmlich et al. 2011a

D. Li et al. 2011

Syrris et al. 2006a

van Tintelen et al. 2006

Otterspoor et al. 2007

Joshi-Mukherjee et al. 2008

\begin{tabular}{|c|c|c|}
\hline \multirow[t]{2}{*}{ Protein } & \multicolumn{2}{|c|}{ References presented in alphabetical and year order } \\
\hline & Tandri et al. 2008 & Nakajima et al. 2011 \\
\hline & Bhuiyan et al. 2009 & $\begin{array}{l}\text { Ostrowska Dahlgren } \\
\text { et al. } 2011\end{array}$ \\
\hline & den Haan et al. 2009 & Pamuru et al. 2011 \\
\hline & Fidler et al. 2009 & Paul et al. 2011 \\
\hline & $\begin{array}{l}\text { Hall et al. } 2009 \\
\text { Oiu et al. } 2009\end{array}$ & $\begin{array}{l}\text { van der Zwaag et al. } \\
\text { 2011a }\end{array}$ \\
\hline & $\begin{array}{l}\text { Watkins et al. } 2009 \\
\text { Wu et al. } 2009\end{array}$ & Zhang et al. 2012 \\
\hline & \multirow{4}{*}{\multicolumn{2}{|c|}{$\begin{array}{l}\text { Christensen et al. } 2010 \mathrm{a} \\
\text { Christensen et al. } 2010 \mathrm{~b} \\
\text { Cox et al. } 2010 \\
\text { Xu et al. } 2010\end{array}$}} \\
\hline & & \\
\hline & & \\
\hline & & \\
\hline & \multirow{6}{*}{\multicolumn{2}{|c|}{$\begin{array}{l}\text { Aneq et al. } 2011 \\
\text { Adachi and Isobe } 2011 \\
\text { Gandjbakhch et al. } 2011 \\
\text { Gehmlich et al. } 2011 \mathrm{a} \\
\text { Kapplinger et al. } 2011 \\
\text { Larsen et al. } 2011\end{array}$}} \\
\hline & & \\
\hline & & \\
\hline & & \\
\hline & & \\
\hline & & \\
\hline
\end{tabular}

Lahtinen et al. 2008
Ryanodine receptor 2 (RYR2)

\section{Rampazzo et al. 1995}

Tiso et al. 2001

Milting et al. 2006

Koop et al. 2008

Desmin

Klauke et al. 2010

Otten et al. 2010

van Spaendonck-Zwarts et al. 2010

McLendon and Robbins 2011

Ram and Van Wagoner 2008
Lamin $\mathrm{A} / \mathrm{C}$

Striatin

Titin

TGF $\beta 3$
Quarta et al. 2011

Meurs et al. 2010

Taylor et al. 2011

Beffagna et al. 2005 
Table 3 Animal models

References presented in alphabetical and year order

\begin{tabular}{ll}
\hline Bierkamp et al. 1996 & Martin et al. 2009 \\
Ruiz et al. 1996 & McCauley and Wehrens 2009 \\
& Pilichou et al. 2009
\end{tabular}

Gallicano et al. 2001

Meurs et al. 2010

Grossmann et al. 2004

Kirchhof et al. 2006

Meurs et al. 2006

Pilichou et al. 2006

Meurs et al. 2007

Oxford et al. 2007

Oyama et al. 2008

Table 4 Reviews, commentaries, collections and comparative studies published since 2000 reporting that certain mutations in human genes encoding desmosomal proteins and glycoproteins contribute to arrhythmogenic ventricular cardiomyopathies (ARVC) or Naxos and Carvajal diseases

References presented in alphabetical and year order
Corrado et al. 2000

Marcus 2000

Corrado et al. 2001

Franz et al. 2001

Gemayel et al. 2001

McRae et al. 2001

Kimura et al. 2002

Lee et al. 2002

Wichter et al. 2002

\section{Ahmad 2003}

Bluemke et al. 2003

di Cesare 2003

Paul et al. 2003

Casolo et al. 2004

Protonotarios and

Tsatsopoulou 2004

Tomé Esteban et al. 2004

Basso and Thiene 2005

Dokuparti et al. 2005

Haverkamp et al. 2005
Navarcikova et al. 2005

Schultheiss et al. 2005

Sen-Chowdhry et al. 2005

Thiene et al. 2005

Wichter et al. 2005

Basso et al. 2006

Calabrese et al. 2006

MacRae et al. 2006

Rampazzo 2006

Sen-Chowdhry and McKenna 2006

Tsatsopoulou et al. 2006

Bazzi and Christiano 2007

Marcus et al. 2007

Pereira et al. 2007

Sen-Chowdhry et al. 2007a

Sen-Chowdhry et al. 2007b

Thiene et al. 2007

van Tintelen et al. 2007

Awad et al. 2008

Inama et al. 2008

Whyte et al. 2008
Table 4 (continued)

References presented in alphabetical and year order

Asimaki et al. 2009

Bolling and Jonkman 2009

Corrado et al. 2009

El Demellawy et al. 2009

Gandjbakhch et al. 2009

Herren et al. 2009

Mackey-Bojack et al. 2009

Marcus and Zareba 2009

McCauley and Wehrens $2009^{\mathrm{a}}$

Saffitz 2009

Saffitz et al. 2009

Sheikh et al. 2009

Andrews et al. 2010

Barahona-Dussault et al. 2010

Bauce et al. 2010

Delmar and McKenna 2010

Elger et al. 2010

Ellinor et al. 2010

Fressart et al. 2010

J. Li and Radice 2010

Lombardi and Marian 2010

Marcus et al. 2010

Migliore et al. 2010

Murphy et al. 2010

Neuber et al. 2010

Pieperhoff et al. 2010

Remme and Bezzina 2010

Roberts et al. 2010

Sen-Chowdhry et al. 2010a

Xu et al. 2010

Asimaki and Saffitz 2011

Asimaki et al. 2011

Avramides et al. 2011

Azaouagh et al. 2011

Basso et al. 2011

Corrado et al. 2011

Ghosh and Haddad 2011

Lahtinen et al. 2011

Lombardi and Marian 2011

Mezzano and Sheikh 2011

Palmisano et al. 2011

Smith 2011

van der Zwaag et al. 2011b

Pieperhoff 2012
Maass 2010

Hamilton 2009

van der Zwaag et al. $2009^{\mathrm{b}}$

${ }^{a}$ Animal model review

${ }^{\mathrm{b}}$ This review contains information on a database from clinical research and other types of data on variants in genes causing arrhythmogenic right ventricular dysplasia/cardiomyopathy (ARVD/C)

Acknowledgement We thank Werner W. Franke for his encouragement, critical comments and many discussions.

Open Access This article is distributed under the terms of the Creative Commons Attribution License which permits any use, distribution, and reproduction in any medium, provided the original author(s) and the source are credited.

\section{References}

Adachi S, Isobe M (2011) Plakophilin-2 gene could be a causative factor in arrhythmogenic right ventricular cardiomyopathy. Circ J $76: 51-52$

Ahmad F (2003) The molecular genetics of arrhythmogenic right ventricular dysplasia-cardiomyopathy. Clin Invest Med 26:167178

Alcalai R, Metzger S, Rosenheck S, Meiner V, Chajek-Shaul T (2003) A recessive mutation in desmoplakin causes arrhythmogenic right 
ventricular dysplasia, skin disorder, and woolly hair. J Am Coll Cardiol 42:319-327

Andrews TD, Cook SS, Baumeister M, Hickey KT (2010) ARVC: help prevent sudden death. Nurse Pract 35:26-33

Aneq MA, Fluur C, Rehnberg M, Soderkvist P, Engvall J, Nylander E, Gunnarsson C (2011) Novel plakophilin-2 mutation: three-generation family with arrhythmogenic right ventricular cardiomyopathy. Scand Cardiovasc J doi:10.3109/14017431.14012011.14636068

Antoniades L, Tsatsopoulou A, Anastasakis A, Syrris P, Asimaki A, Panagiotakos D, Zambartas C, Stefanadis C, McKenna WJ, Protonotarios N (2006) Arrhythmogenic right ventricular cardiomyopathy caused by deletions in plakophilin-2 and plakoglobin (Naxos disease) in families from Greece and Cyprus: genotype-phenotype relations, diagnostic features and prognosis. Eur Heart J 27:22082216

Asimaki A, Saffitz JE (2011) The role of endomyocardial biopsy in ARVC: looking beyond histology in search of new diagnostic markers. J Cardiovasc Electrophysiol 22:111-117

Asimaki A, Syrris P, Wichter T, Matthias P, Saffitz JE, McKenna WJ (2007) A novel dominant mutation in plakoglobin causes arrhythmogenic right ventricular cardiomyopathy. Am J Hum Genet 81:964-973

Asimaki A, Tandri H, Huang H, Halushka MK, Gautam S, Basso C, Thiene G, Tsatsopoulou A, Protonotarios N, McKenna WJ, Calkins H, Saffitz JE (2009) A new diagnostic test for arrhythmogenic right ventricular cardiomyopathy. N Engl J Med 360:10751084

Asimaki A, Tandri H, Duffy ER, Winterfield JR, Mackey-Bojack S, Picken MM, Cooper LT, Wilber DJ, Marcus FI, Basso C, Thiene G, Tsatsopoulou A, Protonotarios N, Stevenson WG, McKenna WJ, Gautam S, Remick DG, Calkins H, Saffitz JE (2011) Altered desmosomal proteins in granulomatous myocarditis and potential pathogenic links to arrhythmogenic right ventricular cardiomyopathy. Circ Arrhythm Electrophysiol 4:743-752

Avramides D, Protonotarios N, Asimaki A, Matsakas E (2011) Arrhythmogenic right ventricular cardiomyopathy/dysplasia. Hellenic J Cardiol 52:452-461

Awad MM, Dalal D, Cho E, Amat-Alarcon N, James C, Tichnell C, Tucker A, Russell SD, Bluemke DA, Dietz HC, Calkins H, Judge DP (2006a) DSG2 mutations contribute to arrhythmogenic right ventricular dysplasia/cardiomyopathy. Am J Hum Genet 79:136142

Awad MM, Dalal D, Tichnell C, James C, Tucker A, Abraham T, Spevak PJ, Calkins H, Judge DP (2006b) Recessive arrhythmogenic right ventricular dysplasia due to novel cryptic splice mutation in PKP2. Hum Mutat 27:1157

Awad MM, Calkins H, Judge DP (2008) Mechanisms of disease: molecular genetics of arrhythmogenic right ventricular dysplasia/cardiomyopathy. Nat Clin Pract Cardiovasc Med 5:258-267

Azaouagh A, Churzidse S, Konorza T, Erbel R (2011) Arrhythmogenic right ventricular cardiomyopathy/dysplasia: a review and update. Clin Res Cardiol 100:383-394

Barahona-Dussault C, Benito B, Campuzano O, Iglesias A, Leung TL, Robb L, Talajic M, Brugada R (2010) Role of genetic testing in arrhythmogenic right ventricular cardiomyopathy/dysplasia. Clin Genet 77:37-48

Basso C, Thiene G (2005) Adipositas cordis, fatty infiltration of the right ventricle, and arrhythmogenic right ventricular cardiomyopathy. Just a matter of fat? Cardiovasc Pathol 14:37-41

Basso C, Czarnowska E, Barbera MD, Bauce B, Beffagna G, Wlodarska EK, Pilichou K, Ramondo A, Lorenzon A, Wozniek O, Corrado D, Daliento L, Danieli GA, Valente M, Nava A, Thiene G, Rampazzo A (2006) Ultrastructural evidence of intercalated disc remodelling in arrhythmogenic right ventricular cardiomyopathy: an electron microscopy investigation on endomyocardial biopsies. Eur Heart J 27:1847-1854
Basso C, Bauce B, Corrado D, Thiene G (2011) Pathophysiology of arrhythmogenic cardiomyopathy. Nat Rev Cardiol doi:10.1038/ nrcardio.2011.1173

Bauce B, Basso C, Rampazzo A, Beffagna G, Daliento L, Frigo G, Malacrida S, Settimo L, Danieli G, Thiene G, Nava A (2005) Clinical profile of four families with arrhythmogenic right ventricular cardiomyopathy caused by dominant desmoplakin mutations. Eur Heart J 26:1666-1675

Bauce B, Nava A, Beffagna G, Basso C, Lorenzon A, Smaniotto G, De Bortoli M, Rigato I, Mazzotti E, Steriotis A, Marra MP, Towbin JA, Thiene G, Danieli GA, Rampazzo A (2010) Multiple mutations in desmosomal proteins encoding genes in arrhythmogenic right ventricular cardiomyopathy/dysplasia. Heart Rhythm 7:22-29

Bazzi H, Christiano AM (2007) Broken hearts, woolly hair, and tattered skin: when desmosomal adhesion goes awry. Curr Opin Cell Biol 19:515-520

Beffagna G, Occhi G, Nava A, Vitiello L, Ditadi A, Basso C, Bauce B, Carraro G, Thiene G, Towbin JA, Danieli GA, Rampazzo A (2005) Regulatory mutations in transforming growth factor-beta3 gene cause arrhythmogenic right ventricular cardiomyopathy type 1 . Cardiovasc Res 65:366-373

Beffagna G, De Bortoli M, Nava A, Salamon M, Lorenzon A, Zaccolo M, Mancuso L, Sigalotti L, Bauce B, Occhi G, Basso C, Lanfranchi G, Towbin JA, Thiene G, Danieli GA, Rampazzo A (2007) Missense mutations in desmocollin-2N-terminus, associated with arrhythmogenic right ventricular cardiomyopathy, affect intracellular localization of desmocollin-2 in vitro. BMC Med Genet 8:65

Bhuiyan ZA, Jongbloed JD, Smagt J van der, Lombardi PM, Wiesfeld AC, Nelen M, Schouten M, Jongbloed R, Cox MG, Wolferen M van, Rodriguez LM, Gelder IC van, Bikker H, Suurmeijer AJ, Berg MP van den, Mannens MM, Hauer RN, Wilde AA, Tintelen JP van (2009) Desmoglein-2 and desmocollin-2 mutations in Dutch arrhythmogenic right ventricular dysplasia/cardiomypathy patients: results from a multicenter study. Circ Cardiovasc Genet $2: 418-427$

Bierkamp C, McLaughlin KJ, Schwarz H, Huber O, Kemler R (1996) Embryonic heart and skin defects in mice lacking plakoglobin. Dev Biol 180:780-785

Bluemke DA, Krupinski EA, Ovitt T, Gear K, Unger E, Axel L, Boxt LM, Casolo G, Ferrari VA, Funaki B, Globits S, Higgins CB, Julsrud P, Lipton M, Mawson J, Nygren A, Pennell DJ, Stillman A, White RD, Wichter T, Marcus F (2003) MR imaging of arrhythmogenic right ventricular cardiomyopathy: morphologic findings and interobserver reliability. Cardiology 99:153-162

Bolling MC, Jonkman MF (2009) Skin and heart: une liaison dangereuse. Exp Dermatol 18:658-668

Bolling MC, Veenstra MJ, Jonkman MF, Diercks GF, Curry CJ, Fisher J, Pas HH, Bruckner AL (2010) Lethal acantholytic epidermolysis bullosa due to a novel homozygous deletion in DSP: expanding the phenotype and implications for desmoplakin function in skin and heart. Br J Dermatol 162:1388-1394

Bortoli M de, Beffagna G, Bauce B, Lorenzon A, Smaniotto G, Rigato I, Calore M, Li Mura IE, Basso C, Thiene G, Lanfranchi G, Danieli GA, Nava A, Rampazzo A (2010) The p.A897KfsX4 frameshift variation in desmocollin-2 is not a causative mutation in arrhythmogenic right ventricular cardiomyopathy. Eur J Hum Genet 18:776-782

Calabrese F, Basso C, Carturan E, Valente M, Thiene G (2006) Arrhythmogenic right ventricular cardiomyopathy/dysplasia: is there a role for viruses? Cardiovasc Pathol 15:11-17

Calkins HM (2006) Arrhythmogenic right-ventricular dysplasia/ cardiomyopathy. Curr Opin Cardiol 21:55-63

Casolo G, Di Cesare E, Molinari G, Knoll P, Midiri M, Fedele F, Biasi S, Rega L, Casolo F, Barsotti AB (2004) Diagnostic work-up of 
arrhythmogenic right ventricular cardiomyopathy by cardiovascular magnetic resonance. Ital Heart J 5:69-79

Cesare E di (2003) MRI assessment of right ventricular dysplasia. Eur Radiol 13:1387-1393

Christensen AH, Benn M, Bundgaard H, Tybjaerg-Hansen A, Haunso S, Svendsen JH (2010a) Wide spectrum of desmosomal mutations in Danish patients with arrhythmogenic right ventricular cardiomyopathy. J Med Genet 47:736-744

Christensen AH, Benn M, Tybjaerg-Hansen A, Haunso S, Svendsen $\mathrm{JH}(2010 \mathrm{~b})$ Missense variants in plakophilin-2 in arrhythmogenic right ventricular cardiomyopathy patients - disease-causing or innocent bystanders? Cardiology 115:148-154

Christensen AH, Andersen CB, Tybjaerg-Hansen A, Haunso S, Svendsen JH (2011) Mutation analysis and evaluation of the cardiac localization of TMEM43 in arrhythmogenic right ventricular cardiomyopathy. Clin Genet 80:256-264

Corrado D, Buja G, Basso C, Thiene G (2000) Clinical diagnosis and management strategies in arrhythmogenic right ventricular cardiomyopathy. J Electrocardiol 33 (Suppl):49-55

Corrado D, Basso C, Nava A, Thiene G (2001) Arrhythmogenic right ventricular cardiomyopathy: current diagnostic and management strategies. Cardiol Rev 9:259-265

Corrado D, Basso C, Thiene G (2009) Arrhythmogenic right ventricular cardiomyopathy: an update. Heart 95:766-773

Corrado D, Basso C, Pilichou K, Thiene G (2011) Molecular biology and clinical management of arrhythmogenic right ventricular cardiomyopathy/dysplasia. Heart 97:530-539

Cox MG, Smagt JJ van der, Noorman M, Wiesfeld AC, Volders PG, Langen IM van, Atsma DE, Dooijes D, Houweling AC, Loh P, Jordaens L, Arens Y, Cramer MJ, Doevendans PA, Tintelen JP van, Wilde AA, Hauer RN (2010) Arrhythmogenic right ventricular dysplasia/cardiomyopathy diagnostic task force criteria: impact of new task force criteria. Circ Arrhythm Electrophysiol 3:126-133

Dalal D, Molin LH, Piccini J, Tichnell C, James C, Bomma C, Prakasa K, Towbin JA, Marcus FI, Spevak PJ, Bluemke DA, Abraham T, Russell SD, Calkins H, Judge DP (2006) Clinical features of arrhythmogenic right ventricular dysplasia/cardiomyopathy associated with mutations in plakophilin-2. Circulation 113:1641-1649

Delmar M, McKenna WJ (2010) The cardiac desmosome and arrhythmogenic cardiomyopathies: from gene to disease. Circ Res 107:700-714

Dokuparti MV, Pamuru PR, Thakkar B, Tanjore RR, Nallari P (2005) Etiopathogenesis of arrhythmogenic right ventricular cardiomyopathy. J Hum Genet 50:375-381

El Demellawy D, Nasr A, Alowami S (2009) An updated review on the clinicopathologic aspects of arrhythmogenic right ventricular cardiomyopathy. Am J Forensic Med Pathol 30:78-83

Elger BS, Michaud K, Fellmann F, Mangin P (2010) Sudden death: ethical and legal problems of post-mortem forensic genetic testing for hereditary cardiac diseases. Clin Genet 77:287-292

Ellinor PT, MacRae CA, Thierfelder L (2010) Arrhythmogenic right ventricular cardiomyopathy. Heart Fail Clin 6:161-177

Fabritz L, Hoogendijk MG, Scicluna BP, Amersfoorth SC van, Fortmueller L, Wolf S, Laakmann S, Kreienkamp N, Piccini I, Breithardt G, Noppinger PR, Witt H, Ebnet K, Wichter T, Levkau B, Franke WW, Pieperhoff S, Bakker JM de, Coronel R, Kirchhof P (2011) Load-reducing therapy prevents development of arrhythmogenic right ventricular cardiomyopathy in plakoglobin-deficient mice. J Am Coll Cardiol 57:740-750

Fidler LM, Wilson GJ, Liu F, Cui X, Scherer SW, Taylor GP, Hamilton RM (2009) Abnormal connexin43 in arrhythmogenic right ventricular cardiomyopathy caused by plakophilin-2 mutations. J Cell Mol Med 13:4219-4228

Franz WM, Mueller OJ, Katus HA (2001) Cardiomyopathies: from genetics to the prospect of treatment. Lancet 358:1627-1637
Fressart V, Duthoit G, Donal E, Probst V, Deharo JC, Chevalier P, Klug D, Dubourg O, Delacretaz E, Cosnay P, Scanu P, Extramiana F, Keller D, Hidden-Lucet F, Simon F, Bessirard V, Roux-Buisson N, Hebert JL, Azarine A, Casset-Senon D, Rouzet F, Lecarpentier Y, Fontaine G, Coirault C, Frank R, Hainque B, Charron P (2010) Desmosomal gene analysis in arrhythmogenic right ventricular dysplasia/cardiomyopathy: spectrum of mutations and clinical impact in practice. Europace $12: 861-868$

Gallicano GI, Bauer C, Fuchs E (2001) Rescuing desmoplakin function in extra-embryonic ectoderm reveals the importance of this protein in embryonic heart, neuroepithelium, skin and vasculature. Development 128:929-941

Gandjbakhch E, Fressart V, Bertaux G, Faivre L, Simon F, Frank R, Fontaine G, Villard E, Coirault C, Hainque B, Charron P (2009) Sporadic arrhythmogenic right ventricular cardiomyopathy/dysplasia due to a de novo mutation. Europace 11:379-381

Gandjbakhch E, Charron P, Fressart V, Lorin de la Grandmaison G, Simon F, Gary F, Vite A, Hainque B, Hidden-Lucet F, Komajda M, Villard E (2011) Plakophilin 2A is the dominant isoform in human heart tissue: consequences for the genetic screening of arrhythmogenic right ventricular cardiomyopathy. Heart 97:844849

Garcia-Gras E, Lombardi R, Giocondo MJ, Willerson JT, Schneider MD, Khoury DS, Marian AJ (2006) Suppression of canonical Wnt/beta-catenin signaling by nuclear plakoglobin recapitulates phenotype of arrhythmogenic right ventricular cardiomyopathy. J Clin Invest 116:2012-2021

Gehmlich K, Asimaki A, Cahill TJ, Ehler E, Syrris P, Zachara E, Re F, Avella A, Monserrat L, Saffitz JE, McKenna WJ (2010) Novel missense mutations in exon 15 of desmoglein-2: role of the intracellular cadherin segment in arrhythmogenic right ventricular cardiomyopathy? Heart Rhythm 7:1446-1453

Gehmlich K, Syrris P, Peskett E, Evans A, Ehler E, Asimaki A, Anastasakis A, Tsatsopoulou A, Vouliotis AI, Stefanadis C, Saffitz JE, Protonotarios N, McKenna WJ (2011a) Mechanistic insights into arrhythmogenic right ventricular cardiomyopathy caused by desmocollin-2 mutations. Cardiovasc Res 90:77-87

Gehmlich K, Syrris P, Reimann M, Asimaki A, Ehler E, Evans A, Quarta G, Pantazis A, Saffitz JE, McKenna WJ (2011b) Molecular changes in the heart of a severe case of arrhythmogenic right ventricular cardiomyopathy caused by a desmoglein- 2 null allele. Cardiovasc Pathol. doi:10.1016/j.carpath.2011.1009.1005

Gemayel C, Pelliccia A, Thompson PD (2001) Arrhythmogenic right ventricular cardiomyopathy. J Am Coll Cardiol 38:1773-1781

Gerull B, Heuser A, Wichter T, Paul M, Basson CT, McDermott DA, Lerman BB, Markowitz SM, Ellinor PT, MacRae CA, Peters S, Grossmann KS, Drenckhahn J, Michely B, Sasse-Klaassen S, Birchmeier W, Dietz R, Breithardt G, Schulze-Bahr E, Thierfelder L (2004) Mutations in the desmosomal protein plakophilin-2 are common in arrhythmogenic right ventricular cardiomyopathy. Nat Genet 36:1162-1164

Ghosh N, Haddad H (2011) Recent progress in the genetics of cardiomyopathy and its role in the clinical evaluation of patients with cardiomyopathy. Curr Opin Cardiol 26:155-164

Gomes J, Finlay M, Ahmed AK, Ciaccio EJ, Asimaki A, Saffitz JE, Quarta G, Nobles M, Syrris P, Chaubey S, McKenna WJ, Tinker A, Lambiase PD (2012) Electrophysiological abnormalities precede overt structural changes in arrhythmogenic right ventricular cardiomyopathy due to mutations in desmoplakin-A combined murine and human study. Eur Heart J. doi:10.1093/eurheartj/ ehr1472

Grossmann KS, Grund C, Huelsken J, Behrend M, Erdmann B, Franke WW, Birchmeier W (2004) Requirement of plakophilin 2 for heart morphogenesis and cardiac junction formation. J Cell Biol 167:149-160 
Haan AD den, Tan BY, Zikusoka MN, Llado LI, Jain R, Daly A, Tichnell C, James C, Amat-Alarcon N, Abraham T, Russell SD, Bluemke DA, Calkins H, Dalal D, Judge DP (2009) Comprehensive desmosome mutation analysis in North Americans with arrhythmogenic right ventricular dysplasia/cardiomyopathy. Circ Cardiovasc Genet 2:428-435

Hall C, Li S, Li H, Creason V, Wahl JK 3rd (2009) Arrhythmogenic right ventricular cardiomyopathy plakophilin-2 mutations disrupt desmosome assembly and stability. Cell Commun Adhes 16:1-13

Hamilton RM (2009) Arrhythmogenic right ventricular cardiomyopathy. Pacing Clin Electrophysiol 32 (Suppl 2):S44-S51

Haverkamp W, Rolf S, Osterziel KJ, Dietz R, Peters S (2005) Arrhythmogenic right ventricular cardiomyopathy. Herz 30:565-570

Herren T, Gerber PA, Duru F (2009) Arrhythmogenic right ventricular cardiomyopathy/dysplasia: a not so rare "disease of the desmosome" with multiple clinical presentations. Clin Res Cardiol 98:141-158

Heuser A, Plovie ER, Ellinor PT, Grossmann KS, Shin JT, Wichter T, Basson CT, Lerman BB, Sasse-Klaassen S, Thierfelder L, MacRae CA, Gerull B (2006) Mutant desmocollin-2 causes arrhythmogenic right ventricular cardiomyopathy. Am J Hum Genet 79:1081-1088

Huang H, Asimaki A, Lo D, McKenna W, Saffitz J (2008) Disparate effects of different mutations in plakoglobin on cell mechanical behavior. Cell Motil Cytoskeleton 65:964-978

Inama G, Pedrinazzi C, Gazzaniga P, Reduzzi C, Donato G, Munoz CV, Pacchioni A, Inama L, Della Frera W, Durin O (2008) Current limitations and new perspectives in the diagnosis of arrhythmogenic right ventricular cardiomyopathy/dysplasia. G Ital Cardiol (Rome) 9:83S-89S

Joshi-Mukherjee R, Coombs W, Musa H, Oxford E, Taffet S, Delmar M (2008) Characterization of the molecular phenotype of two arrhythmogenic right ventricular cardiomyopathy (ARVC)-related plakophilin-2 (PKP2) mutations. Heart Rhythm 5:1715-1723

Kannankeril PJ, Bhuiyan ZA, Darbar D, Mannens MM, Wilde AA, Roden DM (2006) Arrhythmogenic right ventricular cardiomyopathy due to a novel plakophilin 2 mutation: wide spectrum of disease in mutation carriers within a family. Heart Rhythm 3:939-944

Kant S, Krull P, Eisner S, Leube RE, Krusche CA (2012) Histological and ultrastructural abnormalities in murine desmoglein 2-mutant hearts. Cell Tissue Res. doi:10.1007/s00441-011-1322-3

Kaplan SR, Gard JJ, Protonotarios N, Tsatsopoulou A, Spiliopoulou C, Anastasakis A, Squarcioni CP, McKenna WJ, Thiene G, Basso C, Brousse N, Fontaine G, Saffitz JE (2004) Remodeling of myocyte gap junctions in arrhythmogenic right ventricular cardiomyopathy due to a deletion in plakoglobin (Naxos disease). Heart Rhythm 1:3-11

Kapplinger JD, Landstrom AP, Salisbury BA, Callis TE, Pollevick GD, Tester DJ, Cox MG, Bhuiyan Z, Bikker H, Wiesfeld AC, Hauer RN, Tintelen JP van, Jongbloed JD, Calkins H, Judge DP, Wilde AA, Ackerman MJ (2011) Distinguishing arrhythmogenic right ventricular cardiomyopathy/dysplasia-associated mutations from background genetic noise. J Am Coll Cardiol 57:2317-2327

Kimura F, Sakai F, Sakomura Y, Fujimura M, Ueno E, Matsuda N, Kasanuki H, Mitsuhashi N (2002) Helical CT features of arrhythmogenic right ventricular cardiomyopathy. Radiographics 22:1111-1124

Kirchhof P, Fabritz L, Zwiener M, Witt H, Schafers M, Zellerhoff S, Paul M, Athai T, Hiller KH, Baba HA, Breithardt G, Ruiz P, Wichter T, Levkau B (2006) Age- and training-dependent development of arrhythmogenic right ventricular cardiomyopathy in heterozygous plakoglobin-deficient mice. Circulation 114:1799-1806

Klauke B, Kossmann S, Gaertner A, Brand K, Stork I, Brodehl A, Dieding M, Walhorn V, Anselmetti D, Gerdes D, Bohms B, Schulz U, Zu Knyphausen E, Vorgerd M, Gummert J, Milting H (2010) De novo desmin-mutation N116S is associated with arrhythmogenic right ventricular cardiomyopathy. Hum Mol Genet 19:4595-4607

Klauke B, Baecker C, Muesebeck J, Schulze-Bahr E, Gerdes D, Gaertner A, Milting H (2011) Deleterious effects of the TMEM43 mutation p.S358L found in a German family with arrhythmogenic right ventricular cardiomyopathy and sudden cardiac death. Cell Tiss Res (this issue)

Koop A, Goldmann P, Chen SR, Thieleczek R, Varsanyi M (2008) ARVC-related mutations in divergent region 3 alter functional properties of the cardiac ryanodine receptor. Biophys J 94:4668-4677

Krusche CA, Holthoefer B, Hofe V, Sandt AM van de, Eshkind L, Bockamp E, Merx MW, Kant S, Windoffer R, Leube RE (2011) Desmoglein 2 mutant mice develop cardiac fibrosis and dilation. Basic Res Cardiol 106:617-633

Lahtinen AM, Lehtonen A, Kaartinen M, Toivonen L, Swan H, Widen E, Lehtonen E, Lehto VP, Kontula K (2008) Plakophilin-2 missense mutations in arrhythmogenic right ventricular cardiomyopathy. Int J Cardiol 126:92-100

Lahtinen AM, Lehtonen E, Marjamaa A, Kaartinen M, Helio T, Porthan K, Oikarinen L, Toivonen L, Swan H, Jula A, Peltonen L, Palotie A, Salomaa V, Kontula K (2011) Population-prevalent desmosomal mutations predisposing to arrhythmogenic right ventricular cardiomyopathy. Heart Rhythm 8:1214-1221

Larsen MK, Nissen PH, Berge KE, Leren TP, Kristensen IB, Jensen HK, Banner J (2011) Molecular autopsy in young sudden cardiac death victims with suspected cardiomyopathy. Forensic Sci Int. doi:10.1016/j.forsciint.2011.1011.1020

Lee KT, Lai WT, Yen HW, Voon WC, Hwang CH, Lu YH, Lin TH, Sheu SH (2002) Arrhythmogenic right ventricular cardiomyopathy: three cases. Kaohsiung J Med Sci 18:523-528

Li J, Radice GL (2010) A new perspective on intercalated disc organization: implications for heart disease. In: Mohoney G, Mueller EJ, Koch PJ (eds) Dermatology research and practice. Desmosomes and desmosomal cadherin function in skin and heart diseases. Advancements in basic and clinical research. Hindawi, LaVergne, article ID 207835

Li D, Liu Y, Maruyama M, Zhu W, Chen H, Zhang W, Reuter S, Lin SF, Haneline LS, Field LJ, Chen PS, Shou W (2011a) Restrictive loss of plakoglobin in cardiomyocytes leads to arrhythmogenic cardiomyopathy. Hum Mol Genet 20:4582-4596

Li J, Swope D, Raess N, Cheng L, Muller EJ, Radice GL (2011b) Cardiac tissue-restricted deletion of plakoglobin results in progressive cardiomyopathy and activation of $\{$ beta $\}$-catenin signaling. Mol Cell Biol 31:1134-1144

Lombardi R, Marian AJ (2010) Arrhythmogenic right ventricular cardiomyopathy is a disease of cardiac stem cells. Curr Opin Cardiol. doi:10.1097/HCO.1090b1013e3283376daf

Lombardi R, Marian AJ (2011) Molecular genetics and pathogenesis of arrhythmogenic right ventricular cardiomyopathy: a disease of cardiac stem cells. Pediatr Cardiol 32:360-365

Lombardi R, Graca Cabreira-Hansen M da, Bell A, Fromm RR, Willerson JT, Marian AJ (2011) Nuclear plakoglobin is essential for differentiation of cardiac progenitor cells to adipocytes in arrhythmogenic right ventricular cardiomyopathy. Circ Res 109:13421353

Maass K (2010) Arrhythmogenic right ventricular cardiomyopathy and desmin: another gene fits the shoe. Heart Rhythm 7:1065-1066

Mackey-Bojack SM, Roe SJ, Titus JL (2009) Sudden death with circumferential subepicardial fibrofatty replacement: left-sided arrhythmogenic ventricular cardiomyopathy. Am J Forensic Med Pathol 30:209-214

MacRae CA, Birchmeier W, Thierfelder L (2006) Arrhythmogenic right ventricular cardiomyopathy: moving toward mechanism. J Clin Invest 116:1825-1828

Mahoney MY, Sadowski S, Brennan D, Pikander P, Saukko P, Wahl J, Aho H, Heikinheimo K, Bruckner-Tuderman L, Fertala A, Peltonen 
J, Uitto J, Peltonen S (2010) Compound heterozygous desmoplakin mutations result in a phenotype with a combination of myocardial, skin, hair, and enamel abnormalities. J Invest Dermatol 130:968-978

Marcus FI (2000) Electrocardiographic features of inherited diseases that predispose to the development of cardiac arrhythmias, long QT syndrome, arrhythmogenic right ventricular cardiomyopathy/dysplasia, and Brugada syndrome. J Electrocardiol 33 (Suppl):1-10

Marcus FI, Zareba W (2009) The electrocardiogram in right ventricular cardiomyopathy/dysplasia. How can the electrocardiogram assist in understanding the pathologic and functional changes of the heart in this disease? J Electrocardiol 42:e131-e135

Marcus FI, Nava A, Thiene G (2007) Arrhythmogenic RV cardiomyopathy/dysplasia recent advances. Springer, Milan

Marcus FI, McKenna WJ, Sherrill D, Basso C, Bauce B, Bluemke DA, Calkins H, Corrado D, Cox MG, Daubert JP, Fontaine G, Gear K, Hauer R, Nava A, Picard MH, Protonotarios N, Saffitz JE, Sanborn DM, Steinberg JS, Tandri H, Thiene G, Towbin JA, Tsatsopoulou A, Wichter T, Zareba W (2010) Diagnosis of arrhythmogenic right ventricular cardiomyopathy/dysplasia: proposed modification of the Task Force Criteria. Eur Heart J 31:806-814

Martin ED, Moriarty MA, Byrnes L, Grealy M (2009) Plakoglobin has both structural and signalling roles in zebrafish development. Dev Biol 327:83-96

McCauley MD, Wehrens XH (2009) Animal models of arrhythmogenic cardiomyopathy. Dis Model Mech 2:563-570

McKoy G, Protonotarios N, Crosby A, Tsatsopoulou A, Anastasakis A, Coonar A, Norman M, Baboonian C, Jeffery S, McKenna WJ (2000) Identification of a deletion in plakoglobin in arrhythmogenic right ventricular cardiomyopathy with palmoplantar keratoderma and woolly hair (Naxos disease). Lancet 355:2119-2124

McLendon PM, Robbins J (2011) Desmin-related cardiomyopathy: an unfolding story. Am J Physiol Heart Circ Physiol 301:H1220 H1228

McRae AT 3rd, Chung MK, Asher CR (2001) Arrhythmogenic right ventricular cardiomyopathy: a cause of sudden death in young people. Cleve Clin J Med 68:459-467

Merner ND, Hodgkinson KA, Haywood AF, Connors S, French VM, Drenckhahn JD, Kupprion C, Ramadanova K, Thierfelder L, McKenna W, Gallagher B, Morris-Larkin L, Bassett AS, Parfrey PS, Young TL (2008) Arrhythmogenic right ventricular cardiomyopathy type 5 is a fully penetrant, lethal arrhythmic disorder caused by a missense mutation in the TMEM43 gene. Am J Hum Genet 82:809-821

Meurs KM, Lacombe VA, Dryburgh K, Fox PR, Reiser PR, Kittleson MD (2006) Differential expression of the cardiac ryanodine receptor in normal and arrhythmogenic right ventricular cardiomyopathy canine hearts. Hum Genet 120:111-118

Meurs KM, Ederer MM, Stern JA (2007) Desmosomal gene evaluation in boxers with arrhythmogenic right ventricular cardiomyopathy. Am J Vet Res 68:1338-1341

Meurs KM, Mauceli E, Lahmers S, Acland GM, White SN, Lindblad-Toh K (2010) Genome-wide association identifies a deletion in the $3^{\prime}$ untranslated region of striatin in a canine model of arrhythmogenic right ventricular cardiomyopathy. Hum Genet 128:315-324

Mezzano V, Sheikh F (2011) Cell-cell junction remodeling in the heart: possible role in cardiac conduction system function and arrhythmias? Life Sci. doi:10.1016/j.lfs.2011.1012.1009

Migliore F, Zorzi A, Silvano M, Rigato I, Basso C, Thiene G, Corrado D (2010) Clinical management of arrhythmogenic right ventricular cardiomyopathy: an update. Curr Pharm Des 16:2918-2928

Milting H, Lukas N, Klauke B, Korfer R, Perrot A, Osterziel KJ, Vogt J, Peters S, Thieleczek R, Varsanyi M (2006) Composite polymorphisms in the ryanodine receptor 2 gene associated with arrhythmogenic right ventricular cardiomyopathy. Cardiovasc Res 71:496-505

Munkholm J, Christensen AH, Svendsen JH, Andersen CB (2012) Usefulness of immunostaining for plakoglobin as a diagnostic marker of arrhythmogenic right ventricular cardiomyopathy. Am J Cardiol 109:272-275

Murphy DT, Shine SC, Cradock A, Galvin JM, Keelan ET, Murray JG (2010) Cardiac MRI in arrhythmogenic right ventricular cardiomyopathy. AJR Am J Roentgenol 194:W299-W306

Nagaoka I, Matsui K, Ueyama T, Kanemoto M, Wu J, Shimizu A, Matsuzaki M, Horie M (2006) Novel mutation of plakophilin-2 associated with arrhythmogenic right ventricular cardiomyopathy. Circ J 70:933-935

Nakajima T, Kaneko Y, Irie T, Takahashi R, Kato T, Iijima T, Iso T, Kurabayashi M (2011) Compound and digenic heterozygosity in desmosome genes as a cause of arrhythmogenic right ventricular cardiomyopathy in Japanese patients. Circ J. doi:10.1253/circj.CJ1211-0927

Navarcikova S, Hatala R, Zlatos L, Hulin I (2005) Arrhythmogenic right ventricular cardiomyopathy/dysplasia. Bratisl Lek Listy 106:257-261

Neuber S, Muehmer M, Wratten D, Koch PJ, Moll R, Schmidt A (2010) The desmosomal plaque proteins of the plakophilin family. In: Mohoney G, Mueller EJ, Koch PJ (eds) Dermatology research and practice. Desmosomes and desmosomal cadherin function in skin and heart diseases. Advancements in basic and clinical research. Hindawi, LaVergne, article ID 101452

Norgett EE, Hatsell SJ, Carvajal-Huerta L, Cabezas JC, Common J, Purkis PE, Whittock N, Leigh IM, Stevens HP, Kelsell DP (2000) Recessive mutation in desmoplakin disrupts desmoplakinintermediate filament interactions and causes dilated cardiomyopathy, woolly hair and keratoderma. Hum Mol Genet 9:2761-2766

Norgett EE, Lucke TW, Bowers B, Munro CS, Leigh IM, Kelsell DP (2006) Early death from cardiomyopathy in a family with autosomal dominant striate palmoplantar keratoderma and woolly hair associated with a novel insertion mutation in desmoplakin. J Invest Dermatol 126:1651-1654

Norman M, Simpson M, Mogensen J, Shaw A, Hughes S, Syrris P, Sen-Chowdhry S, Rowland E, Crosby A, McKenna WJ (2005) Novel mutation in desmoplakin causes arrhythmogenic left ventricular cardiomyopathy. Circulation 112:636-642

Ostrowska Dahlgren B, Allen M, Lindstrom AC, Bjerke M, Blomstrom-Lundqvist C (2011) A novel variant in plakophilin-2 gene detected in a family with arrhythmogenic right ventricular cardiomyopathy. J Interv Card Electrophysiol. doi:10.1007/ s10840-10011-19643-10844

Otten E, Asimaki A, Maass A, Langen IM van, Wal A van der, Jonge $\mathrm{N}$ de, Berg MP van den, Saffitz JE, Wilde AA, Jongbloed JD, Tintelen JP van (2010) Desmin mutations as a cause of right ventricular heart failure affect the intercalated disks. Heart Rhythm 7:1058-1064

Otterspoor LC, Reichert CL, Cramer MJ, Bhuiyan ZA, Wilde AA, Hauer RN (2007) Arrhythmogenic right ventricular cardiomyopathy: asymptomatic to life threatening as illustrated by the cases of two sisters. Neth Heart J 15:348-353

Oxford EM, Everitt M, Coombs W, Fox PR, Kraus M, Gelzer AR, Saffitz J, Taffet SM, Moise NS, Delmar M (2007) Molecular composition of the intercalated disc in a spontaneous canine animal model of arrhythmogenic right ventricular dysplasia/ cardiomyopathy. Heart Rhythm 4:1196-1205

Oyama MA, Reiken S, Lehnart SE, Chittur SV, Meurs KM, Stern J, Marks AR (2008) Arrhythmogenic right ventricular cardiomyopathy in boxer dogs is associated with calstabin2 deficiency. J Vet Cardiol 10:1-10

Palmisano BT, Rottman JN, Wells QS, DiSalvo TG, Hong CC (2011) Familial evaluation for diagnosis of arrhythmogenic right ventricular dysplasia. Cardiology 119:47-53

Pamuru PR, Maithili DV, Mohiuddin K, Calambur N, Nallari P (2011) Novel mutations in arrhythmogenic right ventricular cardiomyopathy from Indian population. Indian J Hum Genet 17:70-76 
Paul M, Schulze-Bahr E, Breithardt G, Wichter T (2003) Genetics of arrhythmogenic right ventricular cardiomyopathy-status quo and future perspectives. Z Kardiol 92:128-136

Paul M, Wichter T, Kies P, Gerss J, Wollmann C, Rahbar K, Eckardt L, Breithardt G, Schober O, Schulze-Bahr E, Schafers M (2011) Cardiac sympathetic dysfunction in genotyped patients with arrhythmogenic right ventricular cardiomyopathy and risk of recurrent ventricular tachyarrhythmias. J Nucl Med 52:1559-1565

Pereira A, Thomas B, Tavares NJ (2007) Magnetic resonance imaging findings in suspected arrhythmogenic right ventricular dysplasia. Rev Port Cardiol 26:247-253

Pieperhoff S (2012) Gene mutations resulting in the development of ARVC/D could affect cells of the cardiac conduction system. Front Physiol 3:22. doi:10.3389/fphys.2012.00022

Pieperhoff S, Barth M, Rickelt S, Franke WW (2010) Desmosomal molecules in and out of adhering junctions: normal and diseased states of epidermal, cardiac and mesenchymally derived cells. In: Mohoney G, Mueller EJ, Koch PJ (eds) Dermatology research and practice. Desmosomes and desmosomal cadherin function in skin and heart diseases. Advancements in basic and clinical research. Hindawi, LaVergne, article ID 139167

Pilichou K, Nava A, Basso C, Beffagna G, Bauce B, Lorenzon A, Frigo G, Vettori A, Valente M, Towbin J, Thiene G, Danieli GA, Rampazzo A (2006) Mutations in desmoglein-2 gene are associated with arrhythmogenic right ventricular cardiomyopathy. Circulation 113:1171-1179

Pilichou K, Remme CA, Basso C, Campian ME, Rizzo S, Barnett P, Scicluna BP, Bauce B, Hoff MJ van den, Bakker JM de, Tan HL, Valente M, Nava A, Wilde AA, Moorman AF, Thiene G, Bezzina CR (2009) Myocyte necrosis underlies progressive myocardial dystrophy in mouse dsg2-related arrhythmogenic right ventricular cardiomyopathy. J Exp Med 206:1787-1802

Pilichou K, Bezzina CR, Thiene G, Basso C (2011) Arrhythmogenic cardiomyopathy: transgenic animal models provide novel insights into disease pathobiology. Circ Cardiovasc Genet 4:318-326

Posch MG, Posch MJ, Perrot A, Dietz R, Ozcelik C (2008) Variations in DSG2: V56M, V158G and V920G are not pathogenic for arrhythmogenic right ventricular dysplasia/cardiomyopathy. Nat Clin Pract Cardiovasc Med 5:E1

Protonotarios N, Tsatsopoulou A (2004) Naxos disease and Carvajal syndrome: cardiocutaneous disorders that highlight the pathogenesis and broaden the spectrum of arrhythmogenic right ventricular cardiomyopathy. Cardiovasc Pathol 13:185-194

Protonotarios N, Tsatsopoulou A, Anastasakis A, Sevdalis E, McKoy G, Stratos K, Gatzoulis K, Tentolouris K, Spiliopoulou C, Panagiotakos D, McKenna W, Toutouzas P (2001) Genotype-phenotype assessment in autosomal recessive arrhythmogenic right ventricular cardiomyopathy (Naxos disease) caused by a deletion in plakoglobin. J Am Coll Cardiol 38:1477-1484

Protonotarios NI, Tsatsopoulou AA, Gatzoulis KA (2002) Arrhythmogenic right ventricular cardiomyopathy caused by a deletion in plakoglobin (Naxos disease). Card Electrophysiol Rev 6:72-80

Qiu X, Liu W, Hu D, Zhu T, Li C, Li L, Guo C, Liu X, Wang L, Zheng H, Wang C, Diao Q, Shi D, Zhan P, Deng Y, Liu K, Wang Y, Liu B, Liu H, Zhang L (2009) Mutations of plakophilin-2 in Chinese with arrhythmogenic right ventricular dysplasia/cardiomyopathy. Am J Cardiol 103:1439-1444

Quarta G, Syrris P, Ashworth M, Jenkins S, Zuborne Alapi K, Morgan J, Muir A, Pantazis A, McKenna WJ, Elliott PM (2011) Mutations in the lamin $\mathrm{A} / \mathrm{C}$ gene mimic arrhythmogenic right ventricular cardiomyopathy. Eur Heart J. doi:10.1093/eurheartj/ehr1451

Ram R, Van Wagoner DR (2008) Plakophilin-2 mutations as a cause of arrhythmogenic right ventricular cardiomyopathy: progress toward linking structural with functional changes. Heart Rhythm 5:1724-1725

Rampazzo A (2006) Genetic bases of arrhythmogenic right ventricular cardiomyopathy. Heart Int 2:17
Rampazzo A, Nava A, Erne P, Eberhard M, Vian E, Slomp P, Tiso N, Thiene G, Danieli GA (1995) A new locus for arrhythmogenic right ventricular cardiomyopathy (ARVD2) maps to chromosome 1q42-q43. Hum Mol Genet 4:2151-2154

Rampazzo A, Nava A, Malacrida S, Beffagna G, Bauce B, Rossi V, Zimbello R, Simionati B, Basso C, Thiene G, Towbin JA, Danieli GA (2002) Mutation in human desmoplakin domain binding to plakoglobin causes a dominant form of arrhythmogenic right ventricular cardiomyopathy. Am J Hum Genet 71:1200-1206

Remme CA, Bezzina CR (2010) Cardiac desmosomal (dys)function and myocyte viability. Cell Cycle 9:1246-1252

Roberts JD, Veinot JP, Rutberg J, Gollob MH (2010) Inherited cardiomyopathies mimicking arrhythmogenic right ventricular cardiomyopathy. Cardiovasc Pathol 19:316-320

Ruiz P, Brinkmann V, Ledermann B, Behrend M, Grund C, Thalhammer C, Vogel F, Birchmeier C, Gunthert U, Franke WW, Birchmeier W (1996) Targeted mutation of plakoglobin in mice reveals essential functions of desmosomes in the embryonic heart. J Cell Biol $135: 215-225$

Saffitz JE (2009) Arrhythmogenic cardiomyopathy and abnormalities of cell-to-cell coupling. Heart Rhythm 6:S62-S65

Saffitz JE, Asimaki A, Huang H (2009) Arrhythmogenic right ventricular cardiomyopathy: new insights into disease mechanisms and diagnosis. J Invest Med 57:861-864

Sato T, Nishio H, Suzuki K (2011) Sudden death during exercise in a juvenile with arrhythmogenic right ventricular cardiomyopathy and desmoglein-2 gene substitution: a case report. Leg Med (Tokyo) 13:298-300

Schultheiss HP, Noutsias M, Kuhl U, Gross U, Lassner D, Poller W, Pauschinger M (2005) Cardiomyopathies II. Hypertrophic cardiomyopathy, restrictive cardiomyopathy, arrhythmogenic right ventricular cardiomyopathy. Internist (Berl) 46:1373-1385

Sen-Chowdhry S, McKenna WJ (2006) Sudden cardiac death in the young: a strategy for prevention by targeted evaluation. Cardiology 105:196-206

Sen-Chowdhry S, Syrris P, McKenna WJ (2005) Genetics of right ventricular cardiomyopathy. J Cardiovasc Electrophysiol 16:927-935

Sen-Chowdhry S, Syrris P, McKenna WJ (2007a) Role of genetic analysis in the management of patients with arrhythmogenic right ventricular dysplasia/cardiomyopathy. J Am Coll Cardiol 50:1813-1821

Sen-Chowdhry S, Syrris P, Ward D, Asimaki A, Sevdalis E, McKenna WJ (2007b) Clinical and genetic characterization of families with arrhythmogenic right ventricular dysplasia/cardiomyopathy provides novel insights into patterns of disease expression. Circulation 115:1710-1720

Sen-Chowdhry S, Morgan RD, Chambers JC, McKenna WJ (2010a) Arrhythmogenic cardiomyopathy: etiology, diagnosis, and treatment. Annu Rev Med 61:233-253

Sen-Chowdhry S, Syrris P, Pantazis A, Quarta G, McKenna WJ, Chambers JC (2010b) Mutational heterogeneity, modifier genes, and environmental influences contribute to phenotypic diversity of arrhythmogenic cardiomyopathy. Circ Cardiovasc Genet 3:323-330

Sheikh F, Ross RS, Chen J (2009) Cell-cell connection to cardiac disease. Trends Cardiovasc Med 19:182-190

Simpson MA, Mansour S, Ahnood D, Kalidas K, Patton MA, McKenna WJ, Behr ER, Crosby AH (2009) Homozygous mutation of desmocollin-2 in arrhythmogenic right ventricular cardiomyopathy with mild palmoplantar keratoderma and woolly hair. Cardiology 113:28-34

Smith W (2011) Guidelines for the diagnosis and management of arrhythmogenic right ventricular cardiomyopathy. Heart Lung Circ 20:757-760

Spaendonck-Zwarts K van, Hessem L van, Jongbloed JD, Walle HE de, Capetanaki Y, Kooi AJ van der, Langen IM van, Berg MP van den, Tintelen JP van (2010) Desmin-related myopathy: a review and meta- 
analysis. Clin Genet Epub. doi:10.1111/j.1399-0004.2010.01512.x, ahead of print

Swope D, Cheng L, Gao E, Li J, Radice GL (2012) Loss of cadherinbinding proteins, beta-catenin and plakoglobin, in the heart leads to gap junction remodelling and arrhythmogenesis. Mol Cell Biol. doi:10.1128/MCB.06188-06111

Syrris P, Ward D, Asimaki A, Sen-Chowdhry S, Ebrahim HY, Evans A, Hitomi N, Norman M, Pantazis A, Shaw AL, Elliott PM, McKenna WJ (2006a) Clinical expression of plakophilin-2 mutations in familial arrhythmogenic right ventricular cardiomyopathy. Circulation 113:356-364

Syrris P, Ward D, Evans A, Asimaki A, Gandjbakhch E, Sen-Chowdhry S, McKenna WJ (2006b) Arrhythmogenic right ventricular dysplasia/cardiomyopathy associated with mutations in the desmosomal gene desmocollin-2. Am J Hum Genet 79:978-984

Syrris P, Ward D, Asimaki A, Evans A, Sen-Chowdhry S, Hughes SE, McKenna WJ (2007) Desmoglein-2 mutations in arrhythmogenic right ventricular cardiomyopathy: a genotype-phenotype characterization of familial disease. Eur Heart J 28:581-588

Tandri H, Asimaki A, Dalal D, Saffitz JE, Halushka MK, Calkins H (2008) Gap junction remodeling in a case of arrhythmogenic right ventricular dysplasia due to plakophilin-2 mutation. J Cardiovasc Electrophysiol 19:1212-1214

Taylor M, Graw S, Sinagra G, Barnes C, Slavov D, Brun F, Pinamonti B, Salcedo EE, Sauer W, Pyxaras S, Anderson B, Simon B, Bogomolovas J, Labeit S, Granzier H, Mestroni L (2011) Genetic variation in titin in arrhythmogenic right ventricular cardiomyopathyoverlap syndromes. Circulation 124:876-885

Thiene G, Basso C, Calabrese F, Angelini A, Valente M (2005) Twenty years of progress and beckoning frontiers in cardiovascular pathology: cardiomyopathies. Cardiovasc Pathol 14:165-169

Thiene G, Corrado D, Basso C (2007) Arrhythmogenic right ventricular cardiomyopathy/dysplasia. Orphanet J Rare Dis 2:45

Tintelen JP van, Entius MM, Bhuiyan ZA, Jongbloed R, Wiesfeld AC, Wilde AA, Smagt J van der, Boven LG, Mannens MM, Langen IM van, Hofstra RM, Otterspoor LC, Doevendans PA, Rodriguez LM, Gelder IC van, Hauer RN (2006) Plakophilin-2 mutations are the major determinant of familial arrhythmogenic right ventricular dysplasia/cardiomyopathy. Circulation 113:1650-1658

Tintelen JP van, Hofstra RM, Wiesfeld AC, Berg MP van den, Hauer RN, Jongbloed JD (2007) Molecular genetics of arrhythmogenic right ventricular cardiomyopathy: emerging horizon? Curr Opin Cardiol 22:185-192

Tiso N, Stephan DA, Nava A, Bagattin A, Devaney JM, Stanchi F, Larderet G, Brahmbhatt B, Brown K, Bauce B, Muriago M, Basso C, Thiene G, Danieli GA, Rampazzo A (2001) Identification of mutations in the cardiac ryanodine receptor gene in families affected with arrhythmogenic right ventricular cardiomyopathy type 2 (ARVD2). Hum Mol Genet 10:189-194

Tomé Esteban MT, García-Pinilla JM, McKenna WJ (2004) Update in arrhythmogenic right ventricular cardiomyopathy: genetic, clinical presentation and risk stratification. Rev Esp Cardiol 57:757767

Tsatsopoulou AA, Protonotarios NI, McKenna WJ (2006) Arrhythmogenic right ventricular dysplasia, a cell adhesion cardiomyopathy: insights into disease pathogenesis from preliminary genotypephenotype assessment. Heart 92:1720-1723

Uzumcu A, Norgett EE, Dindar A, Uyguner O, Nisli K, Kayserili H, Sahin SE, Dupont E, Severs NJ, Leigh IM, Yuksel-Apak M,
Kelsell DP, Wollnik B (2006) Loss of desmoplakin isoform I causes early onset cardiomyopathy and heart failure in a Naxos-like syndrome. J Med Genet 43:e5

Watkins DA, Hendricks N, Shaboodien G, Mbele M, Parker M, Vezi BZ, Latib A, Chin A, Little F, Badri M, Moolman-Smook JC, Okreglicki A, Mayosi BM (2009) Clinical features, survival experience, and profile of plakophilin-2 gene mutations in participants of the arrhythmogenic right ventricular cardiomyopathy registry of South Africa. Heart Rhythm 6:S10-S17

Whyte GP, Stephens N, Senior R, Peters N, O'Hanlon R, Sharma S (2008) Differentiation of RVOT-VT and ARVC in an elite athlete. Med Sci Sports Exerc 40:1357-1361

Wichter T, Schulze-Bahr E, Eckardt L, Paul M, Levkau B, Meyborg M, Schafers M, Haverkamp W, Breithardt G (2002) Molecular mechanisms of inherited ventricular arrhythmias. Herz 27:712-739

Wichter T, Paul TM, Eckardt L, Gerdes P, Kirchhof P, Bocker D, Breithardt G (2005) Arrhythmogenic right ventricular cardiomyopathy. Antiarrhythmic drugs, catheter ablation, or ICD? Herz 30:91-101

Wu SL, Wang PN, Hou YS, Zhang XC, Shan ZX, Yu XY, Deng M (2009) Mutation of plakophilin-2 gene in arrhythmogenic right ventricular cardiomyopathy. Chin Med J (Engl) 122:403-407

Xu T, Yang Z, Vatta M, Rampazzo A, Beffagna G, Pilichou K, Scherer SE, Saffitz J, Kravitz J, Zareba W, Danieli GA, Lorenzon A, Nava A, Bauce B, Thiene G, Basso C, Calkins H, Gear K, Marcus F, Towbin JA (2010) Compound and digenic heterozygosity contributes to arrhythmogenic right ventricular cardiomyopathy. J Am Coll Cardiol 55:587-597

Yang Z, Bowles NE, Scherer SE, Taylor MD, Kearney DL, Ge S, Nadvoretskiy VV, DeFreitas G, Carabello B, Brandon LI, Godsel LM, Green KJ, Saffitz JE, Li H, Danieli GA, Calkins H, Marcus F, Towbin JA (2006) Desmosomal dysfunction due to mutations in desmoplakin causes arrhythmogenic right ventricular dysplasia/ cardiomyopathy. Circ Res 99:646-655

Yu CC, Yu CH, Hsueh CH, Yang CT, Juang JM, Hwang JJ, Lin JL, Lai LP (2008) Arrhythmogenic right ventricular dysplasia: clinical characteristics and identification of novel desmosome gene mutations. J Formos Med Assoc 107:548-558

Zhang M, Tavora F, Oliveira JB, Li L, Franco M, Fowler D, Zhao Z, Burke A (2012) PKP2 mutations in sudden death from arrhythmogenic right ventricular cardiomyopathy (ARVC) and sudden unexpected death with negative autopsy (SUDNA). Circ J 76:189-194

Zwaag PA van der, Jongbloed JD, Berg MP van den, Smagt JJ van der, Jongbloed R, Bikker H, Hofstra RM, Tintelen JP van (2009) A genetic variants database for arrhythmogenic right ventricular dysplasia/cardiomyopathy. Hum Mutat 30:1278-1283

Zwaag PA van der, Cox MG, Werf $\mathrm{C}$ van der, Wiesfeld $\mathrm{AC}$, Jongbloed JD, Dooijes D, Bikker H, Jongbloed R, Suurmeijer AJ, Berg MP van den, Hofstra RM, Hauer RN, Wilde AA, Tintelen JP van (2011a) Recurrent and founder mutations in the Netherlands: plakophilin-2 p.Arg79X mutation causing arrhythmogenic right ventricular cardiomyopathy/dysplasia. Neth Heart J 18:583-591

Zwaag PA van der, Tintelen JP van, Gerbens F, Jongbloed JD, Boven LG, Smagt JJ van der, Roest WP van der, Langen IM van, Bikker H, Hauer RN, Berg MP van den, Hofstra RM, Meerman GJ te (2011b) Haplotype sharing test maps genes for familial cardiomyopathies $(\dagger)$. Clin Genet 79:459-467 